Laboratorio de Envejecimiento, Departamento de Kinesiología; Facultad Ciencias de la Salud, Universidad Católica del Maule. Talca, Chile. aKinesiólogo. ${ }^{\mathrm{b}}$ Magíster en Ciencias. 'Doctor en Gerontología. dLicenciado en Kinesiología. enterna de Kinesiología.

Proyecto SENAMA-UCM "Ejecutores Intermedios" 2010-2011.

Recibido el 23 de noviembre de 2015, aceptado el 9 de marzo de 2016.

Correspondencia a: Eladio Mancilla Solorza Dpto. de Kinesiología, Universidad Católica del Maule, Talca-Chile. Teléfono: 56712203485 Fax: 56712203499 emancill@ucm.cl

\section{Fuerza de prensión manual según edad, género y condición funcional en adultos mayores Chilenos entre 60 y 91 años}

\author{
ELADIO MANCILLA S.$^{\mathrm{a}, \mathrm{b}, \mathrm{c}}$, SARA RAMOS F.e, PABLO MORALES B..$^{\mathrm{a}, \mathrm{d}}$
}

Background: Handgrip strength is an important predictor of functional performance among older adults. Aim: To measure handgrip strength in older adults and relate its values to their clinical functional assessment. Material and Methods: Handgrip strength was measured in 1047 older adults living in the community and aged $71.9 \pm 7$ years ( 740 and 307 females). The values obtained were grouped by age, gender and functional condition. The latter was assessed using a score validated in Chile (Functional Assessment of Older Adults or EFAM), that classifies participants as autonomous without risk, autonomous in risk and in risk of becoming non-autonomous. Results: In women, hand grip strength values were $17.4 \pm 5.6$ and $18.7 \pm 5.7 \mathrm{~kg}$ for left and right hand; figures for men were $30.6 \pm 7.8$ and $31.8 \pm 8.3 \mathrm{~kg}$, respectively. According the functional assessment, the figures for autonomous without risk, autonomous with risk and with risk of becoming non-autonomous participants, were $23.5 \pm 9.7,21.8 \pm$ 9.1 and $19.3 \pm 8.2$ respectively. Conclusions: There is an association between the degrees of functional performance of older people and handgrip strength.

(Rev Med Chile 2016; 144: 598-603)

Key words: Aged; Geriatric Assessment; Hand Strength.

$\mathrm{P}$ royecciones demográficas sugieren que la población adulta mayor (AM) a nivel mundial está aumentando considerablemente ${ }^{1,2}$, fenómeno de envejecimiento poblacional que también se está produciendo en Chile. Todas estas transformaciones demográficas evidencian la necesidad de ajustes profundos en términos de políticas sanitarias y sociales ${ }^{3}$. Bajo este contexto, es relevante conocer que el envejecimiento es un proceso natural que ocurre en los individuos, el cual, genera cambios universales relacionados con la edad ${ }^{4}$ y se caracteriza por la pérdida de capacidades físicas, cognitivas ${ }^{5}$ y funcionales ${ }^{6}$ en los sujetos, disminuyendo su plasticidad de adaptación al entorno.

Si bien envejecer no es sinónimo de déficit funcional, la población AM muestra más riesgo de presentar patologías crónicas y por consiguiente muestra un incremento en la probabilidad de pérdida funcional, asociada a cambios morfológicos y sistémicos ${ }^{7}$. Particularmente, se ha relacionado el envejecimiento con una disminución de masa muscular y asociada a esto, una pérdida de la fuerza muscular ${ }^{8}$ que en algunos casos puede expresarse como estados de disfunción y conllevar a sarcopenia ${ }^{9}$. Este síndrome tiene un origen multifactorial, pues además de generar disfunción contráctil, reduce el número de unidades motoras y atrofia de fibras musculares, es producto de disfunción endocrina y metabólica, inflamación sistémica, modificaciones en la innervación muscular y disminución de la respuesta al estrés, por 
tanto, produce un daño global en el individuo lo que se traduce funcionalmente en un deterioro del rendimiento de motricidad, aumento del riesgo de caída, fragilidad, dependencia y muerte 9

A partir de lo anterior, específicamente la Fuerza de Prensión Manual (FPM) se ha utilizado como indicador de fuerza global, estatus nutricional ${ }^{10}$, mortalidad y como predictor de los cambios en la funcionalidad de los adultos mayores $^{11,12}$. Por consiguiente, la fuerza muscular forma parte importante en el desempeño óptimo de las actividades de la vida diaria y por esta razón, su déficit está fuertemente vinculado al rendimiento funcional del AM. En consecuencia, actualmente en Chile, el EFAM (un instrumento que forma parte del Examen de Medicina Preventiva del Adulto Mayor, EMPAM) corresponde al indicador de pérdida funcional del adulto mayor y que clasifica a los AM en tres categorías: Autovalente Sin Riesgo (ASR); Autovalente Con Riesgo (ACR) y Riesgo de Dependencia (RD) ${ }^{13}$. Por consiguiente, el propósito de esta investigación fue conocer el rendimiento de FPM según género, edad y nivel funcional.

\section{Material y Método}

Estudio descriptivo de corte transversal, que evaluó a un grupo de 1.047 AM, 740 mujeres y 307 hombres entre 60 y 91 años, insertos en la comunidad, pertenecientes a tres Centros de Salud Familiar (CESFAM) de la comuna de Talca, en el marco del proyecto SENAMA-UCM "ejecutores intermedios" 2010-2011. Los AM al momento de asistir al CESFAM, fueron invitados a participar. El presente proyecto fue aprobado por el Comité de Bioética de la Universidad Católica del Maule (UCM) y desarrollado por el Laboratorio de
Envejecimiento de la UCM. Una vez firmado el consentimiento informado, los AM fueron evaluados para el estudio, en el que se consideraron las variables edad, género, nivel funcional y FPM.

A todos los participantes se les realizó fichas individuales, registro de antecedentes personales, mórbidos y farmacológicos. A su vez, para establecer parámetros de seguridad se controló presión arterial, frecuencia cardíaca y respiratoria. Posteriormente, se llevó a cabo una evaluación funcional con $\mathrm{EFAM}^{13}$, y una evaluación de FPM, tanto para la mano derecha como la izquierda ${ }^{14}$.

Para la medición de FPM, se utilizó un dinamómetro hidráulico marca JAMAR ${ }^{\circledR}$ Sammons Preston Inc. ${ }^{15}$. Esta evaluación se lleva a cabo con el AM sentado en una silla, con hombro y antebrazo en posición neutra y codo en 90 grados de flexión. El participante realizaba una fuerza de prensión máxima durante 3 segundos, con reposo de 1 minuto entre cada repetición, realizando dos intentos, donde el mejor de ambos fue el que se utilizó para el estudio.

Para el análisis de datos se utilizó el programa SPSS 18.0 para Windows, donde las variables género, edad y condición funcional según EFAM, asociadas con la prueba de FPM se organizaron por número de sujetos $(\mathrm{n})$, promedios $(\overline{\mathbf{x}})$ y desviación estándar (DS).

\section{Resultados}

Los resultados demográficos de la muestra, media y desviación estándar, se organizaron por medio del género para la edad, FPM y clasificación funcional según EFAM (Tabla 1).

El resumen de los datos de FPM derecha e izquierda, promedio, desviación estándar y número de participantes se organizó por funcionalidad, edad en quinquenios y género (Tablas 2 y 3 ). Asi-

Tabla 1. Resumen demográfico organizado por número de sujetos, edad, funcionalidad y fuerza de prensión manual (FPM) izquierda y derecha, por género, para el total de la muestra

\begin{tabular}{|c|c|c|c|c|c|c|c|c|}
\hline & & \multirow[t]{2}{*}{ n } & \multirow[t]{2}{*}{$\overline{\mathbf{x}}$ Edad } & \multirow[t]{2}{*}{$\overline{\mathbf{x}}$ Fuerza $\mathbf{I}^{\circ}$} & \multirow[t]{2}{*}{$\overline{\mathbf{x}}$ Fuerza $\mathbf{D}^{\circ}$} & \multicolumn{3}{|c|}{$\overline{\mathbf{x}}$ Funcionalidad } \\
\hline & & & & & & ASR & ACR & RD \\
\hline \multirow[t]{3}{*}{ Género } & Mujeres & 740 & $71,07 \pm 6,98$ & $17,41 \pm 5,63$ & $18,69 \pm 5,65$ & $19,07 \pm 6,56$ & $18,11 \pm 5,83$ & $16,43 \pm 5,73$ \\
\hline & Hombres & 307 & $72,70 \pm 6,87$ & $30,59 \pm 7,82$ & $31,84 \pm 8,28$ & $32,64 \pm 7,55$ & $31,77 \pm 8,68$ & $27,29 \pm 7,89$ \\
\hline & Total & 1.047 & $71,89 \pm 7,01$ & $21,28 \pm 8,73$ & $22,47 \pm 8,89$ & $23,51 \pm 9,66$ & $21,84 \pm 9,08$ & $19,27 \pm 8,24$ \\
\hline
\end{tabular}

n: Número de sujetos; $\bar{x}$ : Media; I’: Izquierda; $D^{\circ}$ : Derecha; ASR: Autovalente sin riesgo; ACR: Autovalente con riesgo; RD: Riesgo de dependencia. 
mismo, los resultados de la fuerza manual derecha e izquierda distribuida por quinquenios y género (Figuras la y 1b) muestran una declinación de la media de fuerza para ambas manos según avanza la edad y a su vez, las variables según funcionalidad expresan cambios en la media de fuerza de prensión manual en las tres categorías de clasificación de EFAM (Figuras 2a y $2 b$ ).

Tabla 2. Relación entre la media de fuerza de prensión para mano derecha, género y nivel funcional distribuida por edad en quinquenios, para el total de la muestra. Se presentan media, desviación estándar y número de sujetos

\begin{tabular}{|c|c|c|c|c|c|c|}
\hline FPM Der. & $60-64$ & $65-69$ & 70-74 & 75-79 & $80-84$ & 85 y más \\
\hline \multicolumn{7}{|c|}{ Autovalente sin riesgo } \\
\hline Mujer & $\begin{array}{c}21,27 \pm 5,57 \\
n: 56\end{array}$ & $\begin{array}{c}20,14 \pm 5,98 \\
\text { n: } 49\end{array}$ & $\begin{array}{c}19,63 \pm 5,39 \\
\text { n: } 48\end{array}$ & $\begin{array}{c}18,82 \pm 5,10 \\
\mathrm{n}: 38\end{array}$ & $\begin{array}{c}17,44 \pm 4,77 \\
\mathrm{n}: 16\end{array}$ & $\begin{array}{c}14,00 \pm 5,29 \\
n: 3\end{array}$ \\
\hline Hombre & $\begin{array}{c}35,75 \pm 6,89 \\
n: 16\end{array}$ & $\begin{array}{c}35,83 \pm 8,22 \\
\mathrm{n}: 24\end{array}$ & $\begin{array}{c}33,14 \pm 9,07 \\
\text { n: } 36\end{array}$ & $\begin{array}{c}32,32 \pm 7,09 \\
\text { n: } 22\end{array}$ & $\begin{array}{c}28,00 \pm 5,48 \\
\text { n: } 9\end{array}$ & $\begin{array}{c}25,25 \pm 4,86 \\
n: 4\end{array}$ \\
\hline \multicolumn{7}{|c|}{ Autovalente con riesgo } \\
\hline Mujer & $\begin{array}{c}19,15 \pm 5,87 \\
n: 71\end{array}$ & $\begin{array}{c}19,56 \pm 5,10 \\
\mathrm{n}: 98\end{array}$ & $\begin{array}{c}19,10 \pm 5,45 \\
\text { n: } 82\end{array}$ & $\begin{array}{c}17,45 \pm 4,76 \\
\mathrm{n}: 64\end{array}$ & $\begin{array}{c}16,00 \pm 3,88 \\
\text { n: } 25\end{array}$ & $\begin{array}{c}14,25 \pm 5,29 \\
n: 8\end{array}$ \\
\hline Hombre & $\begin{array}{c}33,63 \pm 8,59 \\
n: 19\end{array}$ & $\begin{array}{c}37,04 \pm 8,57 \\
\text { n: } 28\end{array}$ & $\begin{array}{c}31,12 \pm 8,22 \\
\mathrm{n}: 34\end{array}$ & $\begin{array}{c}30,73 \pm 6,39 \\
\text { n: } 37\end{array}$ & $\begin{array}{c}30,00 \pm 7,02 \\
\text { n: } 16\end{array}$ & $\begin{array}{c}41,00 \\
n: 1\end{array}$ \\
\hline \multicolumn{7}{|c|}{ Riesgo de dependencia } \\
\hline Mujer & $\begin{array}{c}18,56 \pm 6,83 \\
n: 27\end{array}$ & $\begin{array}{c}18,67 \pm 6,16 \\
\mathrm{n}: 24\end{array}$ & $\begin{array}{c}18,92 \pm 6,83 \\
\text { n: } 49\end{array}$ & $\begin{array}{c}16,39 \pm 4,83 \\
n: 33\end{array}$ & $\begin{array}{c}15,74 \pm 5,12 \\
\text { n: } 39\end{array}$ & $\begin{array}{c}13,70 \pm 3,40 \\
\mathrm{n}: 10\end{array}$ \\
\hline Hombre & $\begin{array}{c}34,20 \pm 34 \\
\text { n: } 5\end{array}$ & $\begin{array}{c}29,88 \pm 10,58 \\
\text { n: } 8\end{array}$ & $\begin{array}{c}30,75 \pm 6,05 \\
\text { n: } 12\end{array}$ & $\begin{array}{c}26,90 \pm 6,79 \\
\text { n: } 20\end{array}$ & $\begin{array}{c}22,6 \pm 8,46 \\
\text { n: } 10\end{array}$ & $\begin{array}{c}25,33 \pm 5,75 \\
\text { n: } 6\end{array}$ \\
\hline
\end{tabular}

FPM Der. Fuerza de prensión manual derecha; n: número de sujetos.

Tabla 3. Relación entre la media de Fuerza de prensión para mano izquierda, género y nivel funcional distribuida por edad en quinquenios, para el total de la muestra. Se presentan media, desviación estándar y número de sujetos

\begin{tabular}{|c|c|c|c|c|c|c|}
\hline FPM Izq. & $60-64$ & 65-69 & 70-74 & 75-79 & $80-84$ & 85 y más \\
\hline \multicolumn{7}{|c|}{ Autovalente sin riesgo } \\
\hline Mujer & $\begin{array}{c}20,39 \pm 5,14 \\
\text { n: } 56\end{array}$ & $\begin{array}{c}19,04 \pm 6,82 \\
\text { n: } 49\end{array}$ & $\begin{array}{c}17,46 \pm 5,43 \\
\mathrm{n}: 48\end{array}$ & $\begin{array}{c}16,95 \pm 5,23 \\
\text { n: } 38\end{array}$ & $\begin{array}{c}15,63 \pm 5,18 \\
\mathrm{n}: 16\end{array}$ & $\begin{array}{c}18,35 \pm 5,79 \\
n: 3\end{array}$ \\
\hline Hombre & $\begin{array}{c}35,50 \pm 7,55 \\
\text { n: } 16\end{array}$ & $\begin{array}{c}33,67 \pm 7,32 \\
n: 24\end{array}$ & $\begin{array}{c}32,25 \pm 7,19 \\
\mathrm{n}: 36\end{array}$ & $\begin{array}{c}30,95 \pm 5,24 \\
\mathrm{n}: 22\end{array}$ & $\begin{array}{c}26,89 \pm 5,99 \\
\text { n: } 9\end{array}$ & $\begin{array}{c}26,00 \pm 3,74 \\
n: 4\end{array}$ \\
\hline \multicolumn{7}{|c|}{ Autovalente con riesgo } \\
\hline Mujer & $\begin{array}{c}18,96 \pm 5,45 \\
\text { n: } 71\end{array}$ & $\begin{array}{c}18,44 \pm 4,99 \\
\text { n: } 98\end{array}$ & $\begin{array}{c}17,44 \pm 5,83 \\
n: 82\end{array}$ & $\begin{array}{c}16,42 \pm 4,98 \\
\mathrm{n}: 64\end{array}$ & $\begin{array}{c}15,30 \pm 4,17 \\
\text { n: } 25\end{array}$ & $\begin{array}{c}13,83 \pm 3,99 \\
n: 8\end{array}$ \\
\hline Hombre & $\begin{array}{c}34,95 \pm 6,93 \\
\text { n: } 19\end{array}$ & $\begin{array}{c}33,74 \pm 8,02 \\
\text { n: } 28\end{array}$ & $\begin{array}{c}30,85 \pm 8,18 \\
\text { n: } 34\end{array}$ & $\begin{array}{c}28,92 \pm 6,92 \\
\text { n: } 37\end{array}$ & $\begin{array}{c}27,00 \pm 9,38 \\
\text { n: } 16\end{array}$ & $\begin{array}{c}35,00 \\
\mathrm{n}: 1\end{array}$ \\
\hline \multicolumn{7}{|c|}{ Riesgo de dependencia } \\
\hline Mujer & $\begin{array}{c}16,26 \pm 6,67 \\
\text { n: } 27\end{array}$ & $\begin{array}{c}17,00 \pm 5,99 \\
\text { n: } 24\end{array}$ & $\begin{array}{c}16,94 \pm 6,38 \\
n: 49\end{array}$ & $\begin{array}{c}15,21 \pm 4,87 \\
\mathrm{n}: 33\end{array}$ & $\begin{array}{c}15,33 \pm 4,83 \\
\text { n: } 39\end{array}$ & $\begin{array}{c}12,90 \pm 4,12 \\
\mathrm{n}: 10\end{array}$ \\
\hline Hombre & $\begin{array}{c}33,60 \pm 6,23 \\
\text { n: } 5\end{array}$ & $\begin{array}{c}27,25 \pm 11,65 \\
\text { n: } 8\end{array}$ & $\begin{array}{c}29,92 \pm 4,98 \\
\text { n: } 12\end{array}$ & $\begin{array}{c}25,90 \pm 6,59 \\
\text { n: } 20\end{array}$ & $\begin{array}{c}22,10 \pm 6,62 \\
\mathrm{n}: 10\end{array}$ & $\begin{array}{c}24,50 \pm 3,15 \\
\text { n: } 6\end{array}$ \\
\hline
\end{tabular}

FPM Izq. Fuerza de prensión manual izquierda; n: número de sujetos. 

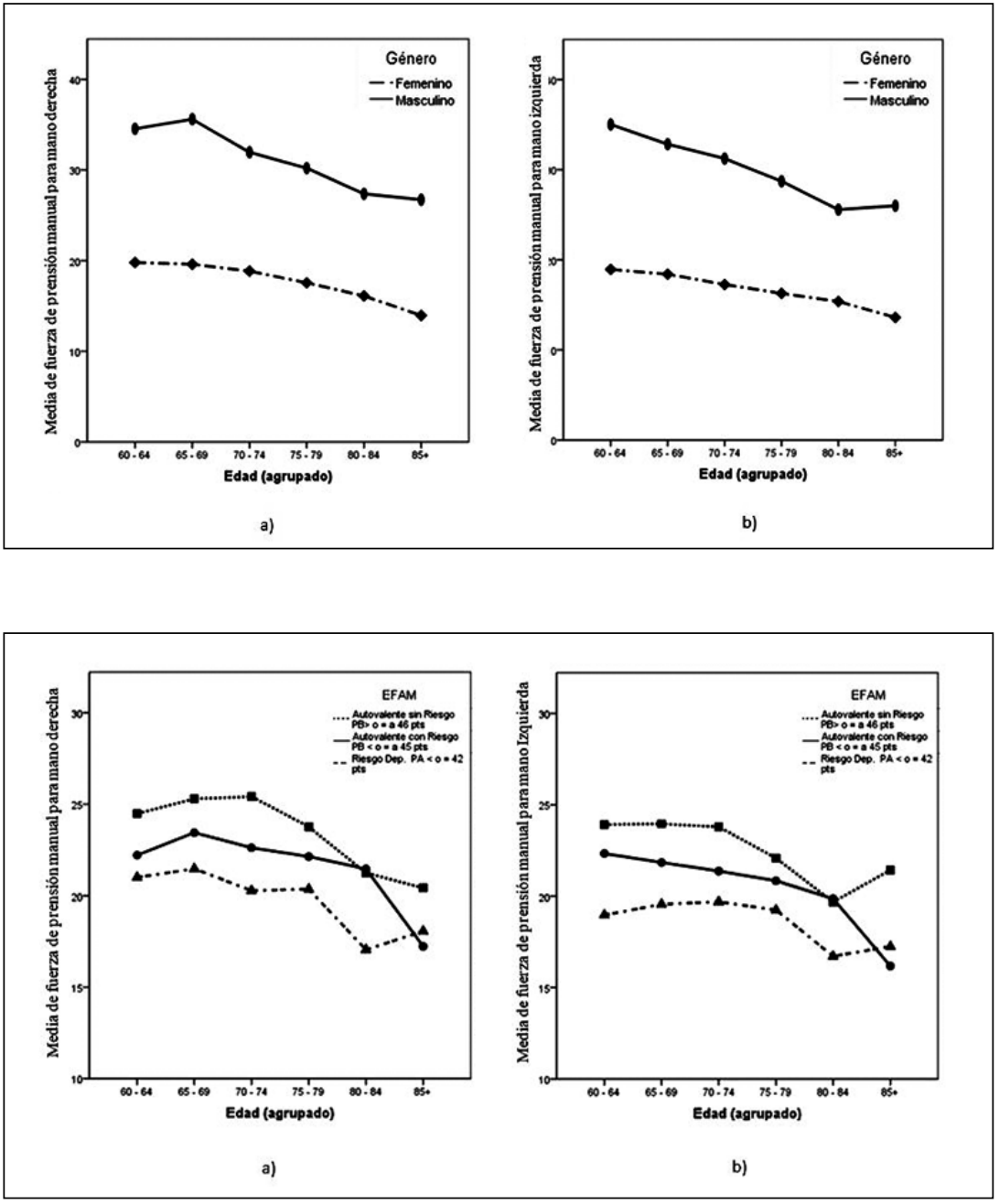

Figura 1. Media de la fuerza de prensión manual agrupada por género y edad en quinquenios; a) mano derecha $y$ b) mano izquierda.
Figura 2. Media de la fuerza de prensión manual agrupada por nivel funcional según EFAM y edad en quinquenios; a) mano derecha y b) mano izquierda.

\section{Discusión}

Los resultados de la muestra del presente estudio distribuidos por edad, permiten observar un descenso en el rendimiento de la prueba de FPM en los AM (Figura 1), lo cual esta de acuerdp con lo demostardo en estudios anteriores ${ }^{16}$. Esto dado que el envejecimiento provoca una disminución general de todas las funciones del organismo ${ }^{8-11}$. En particular luego de los 60 años se produce una pérdida de $20 \%$ de la fuerza de prensión, corolario, entre otros factores, del deterioro de la masa muscular ${ }^{17,18}$. Todo lo anterior ocasiona una disminución en la intensidad y velocidad de contracción muscular ${ }^{19}$. Consecuentemente esto puede llevar a un detrimento en el rendimiento de fuerza muscular o adinamia asociado al envejecimiento y el estrés oxidativo ${ }^{20}$.

En relación al género, se observa una mayor fuerza prensil en hombres con respecto a las mujeres en todos los quinquenios y tanto para la mano derecha (Tabla 2) como la izquierda (Tabla 3). La génesis de estas diferencias comienzan en la etapa de la juventud, período de la vida en la que los hombres van desarrollando mayor masa muscular, condicionado por la actividad física con vínculos sociales que realicen ${ }^{21}$ y la carga androgénica, en la medida que se produce el envejecimiento la masa muscular disminuye para ambos géneros, pero además este proceso se lleva a cabo más tempranamente en las mujeres. En efecto, la media de FPM en el género masculino, a pesar de 
la edad, mantiene un mayor rendimiento respecto a las mujeres ${ }^{10,22}$.

En cuanto al descenso de fuerza que se observa con la edad (Figura 1), es homogéneo tanto en hombres como mujeres. Sin embargo, en los hombres es llamativo el aumento de fuerza posterior a los 85 años, que sobrepasa la media del quinquenio anterior (80-84 años). Esto podría ser explicado porque a esta edad los hombres han sobrepasado su expectativa de vida para la población chilena ${ }^{23}$, ${ }^{24}$. Lo anterior debido a que los han llegado hasta esta etapa del ciclo vital muestran signos de un envejecimiento exitoso ${ }^{25,26}$. En cambio, en las mujeres se puede observar una disminución homogénea en el rendimiento de FPM en todos los quinquenios, probablemente como consecuencia de la pérdida de masa y fuerza muscular; consecuentemente estudios previos han demostrado una relación más intensa entre la pérdida de FPM y la mortalidad en mujeres de edad avanzada ${ }^{25,27-29}$.

En cuanto a la dominancia y su relación con el rendimiento de fuerza manual, es posible observar diferencias entre mano derecha e izquierda (Figura 2), donde la mano derecha obtuvo un mejor rendimiento que la izquierda en todos los grupos, pues, en base a la evidencia la mano derecha tiene más fuerza que la izquierda ${ }^{10}$, no obstante, también está demostrado que no existe una influencia significativa de la dominancia en la FPM ${ }^{10,30}$.

Respecto a la funcionalidad medida con EFAM y su rendimiento de FPM se evidenciaron diferencias entre niveles (Figura 2). Así, el grupo ASR mostró un mejor rendimiento con respecto a ACR y RD, reflejando precisamente la importancia del estatus funcional en la aplicación de fuerza manual como indicador funcional. Sin embargo, en el quinto quinquenio (80-84 años) los grupos ASR y ACR disminuyen sus diferencias, para luego retomarlas en el último quinquenio. Asimismo, en el nivel de RD existe una tendencia a disminuir el rendimiento, a excepción de los AM de 85 y más años, donde se produce un incremento de fuerza, incluso superando la media del quinquenio anterior. Todas estas diferencias pueden ser explicadas puesto que si bien EFAM es una evaluación capaz de clasificar y predecir los deterioros funcionales en el AM, al incorporar variables que abordan los aspectos biopsicosociales que afectan a este grupo etario ${ }^{13}$, no es un test suficientemente sensible para identificar la causa de este deterioro, por lo que en el grupo ACR se pueden observar individuos con un detrimento en un área cognitiva o social, pero no física, manteniendo un óptimo rendimiento en la prueba de FPM y no manifestar una condición de riesgo funcional. Esto producto de la influencia de otras variables, es decir, al utilizar EFAM como instrumento predictor en un grupo numeroso de individuos, no es capaz de discriminar el origen del riesgo funcional que presentan los AM de la muestra estudiada.

Basándonos en los resultados que arrojó el presente estudio, es posible concluir que el rendimiento de los AM en la prueba de FPM mostró un comportamiento relacionado con la edad, género $\mathrm{y}$ nivel funcional. Principalmente, la tendencia observada entre FPM y niveles funcionales, permitiría predecir un comportamiento entre estas dos variables al momento de evaluar un AM. Sobre la base de los antecedentes aportados por la presente investigación y dada la utilidad en la práctica clínica de la prueba de FPM y los aportes que esta ofrece para entender la evolución funcional que puede tener un AM que vive en la comunidad, sería aconsejable analizar la factibilidad de incorporar esta prueba en el examen de medicina preventiva del AM (EMPAM) que se aplica en el sistema de Atención Primaria de Salud (APS) en Chile.

\section{Referencias}

1. Smelser NJ, Baltes PB. International encyclopedia of the social \& behavioral sciences: Elsevier Amsterdam; 2001.

2. Chatterji S, Byles J, Cutler D, Seeman T, Verdes E. Health, functioning, and disability in older adults present status and future implications. Lancet 2015; 385 (9967): 563-75.

3. Medina GP, Mancilla SE, Muñoz CR, Escobar CM. Distancia recorrida y costo fisiológico según el nivel socioeconómico y género durante la prueba de caminata en seis minutos en adultos mayores autovalentes de la ciudad de Talca. Rev Med Chile 2015; 143: 484-92.

4. Chacón RO. Factores asociados a la condición funcional del adulto mayor: Costa Rica 2004-2006.

5. Nelson ME, Layne JE, Bernstein MJ, Nuernberger A, Castaneda C, Kaliton D, et al. The effects of multidimensional home-based exercise on functional performance in elderly people. J Gerontol A Biol Sci Med Sci 2004; 59 (2): 154-60.

6. Nelson ME, Rejeski WJ, Blair SN, Duncan PW, Judge JO, King AC, et al. Physical activity and public health in older adults: recommendation from the American 
College of Sports Medicine and the American Heart Association. Med Sci Sports Exerc 2007; 39 (8): 1435-45.

7. Albala BC, Concha FM, De la Paz GM, Gac EO, Hoyl MT, Marín LP, et al. Manual de Geriatría y Gerontología Para Alumnos http://escuela.med.puc.cl/2005 [cited 2015 19-11-2015]. Available from: http://escuela.med. puc.cl/deptos/programageriatria/PublProgGeriatria. html.

8. Cruz-Jentoft AJ, Baeyens JP, Bauer JM, Boirie Y, Cederholm T, Landi F, et al. Sarcopenia: European consensus on definition and diagnosis: Report of the European Working Group on Sarcopenia in Older People. Age Ageing 2010; 39 (4): 412-23.

9. Kalinkovich A, Livshits G. Sarcopenia-The search for emerging biomarkers. Ageing Res Rev 2015; 22: 58-71.

10. Günther CM, Bürger A, Rickert M, Crispin A, Schulz CU. Grip strength in healthy caucasian adults: reference values. J Hand Surg Am 2008; 33 (4): 558-65.

11. Wang CY, Chen LY. Grip strength in older adults: test-retest reliability and cutoff for subjective weakness of using the hands in heavy tasks. Arch Phys Med Rehabil 2010; 91 (11): 1747-51.

12. Cooper R, Kuh D, Cooper C, Gale CR, Lawlor DA, Matthews F, et al. Objective measures of physical capability and subsequent health: a systematic review. Age Ageing 2011; 40 (1): 14-23.

13. MINSAL. Manual de Aplicación del Examen de Medicina Preventiva del Adulto Mayor. http://web.minsal.cl/ portal/url/item/ab1f81f43ef0c2a6e04001011e011907.pdf Ministerio de Salud Chile; 2001 [updated cited 2015 1911-2015]; Available from: http://web.minsal.cl/portal/ url/item/ab1f81f43ef0c2a6e04001011e011907.pdf

14. Moreira D, Álvarez RRA, DE Gogoy JR, Cambraia AN. Abordagem sobre preensão palmar utilizando o dinamômetro JAMAR ${ }^{\circledR}$ : uma revisão de literatura. Rev Bras Ciênc Mov 2003; 11 (2): 95-9.

15. Muñoz L, De la Vega E, Octavio F, Ortiz B, Lucero K, SEMAC, editors. Fuerza máxima de agarre con mano dominante y no dominante. XV Congreso Internacional de Ergonomía SEMAC; Ciudad Juarez México; 2009.

16. Guede Rojas F, Chirosa Ríos LJ, Vergara Ríos C, Fuentes Contreras J, Delgado Paredes F, Valderrama Campos MJ. Fuerza prensil de mano y su asociación con la edad, género y dominancia de extremidad superior en adultos mayores autovalentes insertos en la comunidad: Un estudio exploratorio. Rev Med Chile 2015; 143: 995-1000.

17. Kaur M. Age-related changes in hand grip strength among rural and urban Haryanvi Jat females. HOMO 2009; 60 (5): 441-50.

18. Leong DP, Teo KK, Rangarajan S, López-Jaramillo P, Avezum A, Orlandini A, et al. Prognostic value of grip strength: findings from the Prospective Urban Rural Epidemiology (PURE) study. Lancet 2015; 386 (9990): 266-73.

19. Aoki H, Demura S. Age differences in hand grip power in the elderly. Archives of gerontology and geriatrics. 2011; 52 (3): e176-e9.

20. Martínez Huenchullán SF, Mancilla Solorza EB. Relación entre proteínas carboniladas y factor necrótico tumoral alfa con fuerza muscular en mujeres jóvenes y mayores: estudio exploratorio. Rev Esp Geriatr Gerontol 2015; 50 (6): 264-9.

21. Kaya RD, Nakazawa M, Hoffman RL, Clark BC. Interrelationship between muscle strength, motor units, and aging. Exp Gerontol 2013; 48 (9): 920-5.

22. Demura S, Aoki H, Sugiura H. Gender differences in hand grip power in the elderly. Arch Gerontol Geriatr 2011; 53 (1): 76-8.

23. Rantanen T, Harris T, Leveille SG, Visser M, Foley D, Masaki K, et al. Muscle strength and body mass index as long-term predictors of mortality in initially healthy men. J Gerontol A Biol Sci Med Sci 2000; 55 (3): M168-73.

24. INE. Proyecciones y estimaciones de población, Total país período información 1950-2050 www.ine.cl: Instituto Nacional de Estadísticas; [cited 2015 19-11-2015]. Available from: http://www.ine.cl/canales/chile_estadistico/demografia_y_vitales/proyecciones/Informes/ MicrosoftWordInforP_T.pdf.

25. Sanchis Sánchez E, Igual Camacho C, Sánchez Frutos J, Blasco Igual MC. Estrategias de envejecimiento activo: revisión bibliográfica. Fisioterapia 2014; 36 (4): 177-86.

26. Yorke AM, Curtis AB, Shoemaker M, Vangsnes E. Grip strength values stratified by age, gender, and chronic disease status in adults aged 50 years and older. J Geriatr Phys Ther 2015; 38 (3): 115-21.

27. Sasaki H, Kasagi F, Yamada M, Fujita S. Grip strength predicts cause-specific mortality in middle-aged and elderly persons. Am J Med 2007; 120 (4): 337-42.

28. Norman K, Stobaus N, González MC, Schulzke JD, Pirlich M. Hand grip strength: outcome predictor and marker of nutritional status. Clin Nutr 2011; 30 (2): 135-42.

29. Daly RM, Rosengren BE, Alwis G, Ahlborg HG, Sernbo I, Karlsson MK. Gender specific age-related changes in bone density, muscle strength and functional performance in the elderly: a-10 year prospective population-based study. BMC Geriatr 2013;13:71.

30. Bohannon RW, Peolsson A, Massy-Westropp N, Desrosiers J, Bear-Lehman J. Reference values for adult grip strength measured with a Jamar dynamometer: a descriptive meta-analysis. Physiotherapy 2006; 92 (1): 11-5. 